\title{
A Novel Transformation Electromagnetic Theory-Based Coverage Optimization Method for Wireless Network
}

\author{
Yuanxuan Li, ${ }^{1}$ Gang Zhu, ${ }^{1}$ Siyu Lin, ${ }^{2}$ Ke Guan, ${ }^{1}$ and Yan $\mathrm{Li}^{1}$ \\ ${ }^{1}$ State Key Laboratory of Rail Traffic Control and Safety, Beijing Jiaotong University, Beijing, China \\ ${ }^{2}$ School of Electronic and Information Engineering, Beijing Jiaotong University, Beijing, China \\ Correspondence should be addressed to Yuanxuan Li; g.liyuanxuan@gmail.com
}

Received 8 July 2013; Accepted 29 September 2013

Academic Editor: Ai Bo

Copyright (c) 2013 Yuanxuan Li et al. This is an open access article distributed under the Creative Commons Attribution License, which permits unrestricted use, distribution, and reproduction in any medium, provided the original work is properly cited.

\begin{abstract}
Improving the reliability of the radio coverage in shadow area is always an important issue for the wireless communications. The emergence of the transformation electromagnetic (TE) technique provides a new method to control the propagation direction of the radio signal. This paper proposes a coverage optimization method based on the TE technique; a cloak which covers the surface of obstacle is designed to improve the coverage performance in shadow area. The material parameters of cloak are calculated by the transformation electromagnetic method. To solve the calculation problem for the rectangular obstacle, the Fourier series is used to approximately describe the rectangular boundaries. The effectiveness of the proposed cloak for the coverage optimization is validated by the theoretical analysis and simulation results. The simulation results show that the coverage performance can be improved significantly.
\end{abstract}

\section{Introduction}

In wireless communication systems, the electromagnetic waves cannot always transmit from the transmission antenna to the receiving antenna directly. When the obstacles block the line-of-sight path, it will bring a significant strength reduction for transmission signal [1]. The signal power may suddenly decrease in the "shadow area," which will lead to the wireless devices losing their connections with the base station [2]. Therefore, improving the coverage quality in shadow area is an important task to ensure the requirement of quality of experience (QoE) of users.

In order to cope with the coverage problem, there are two traditional methods to improve the signal strength in the shadow area: increase the transmission power at the transmitter and deploy the relay nodes for the coverage extension [3]. Several literatures addressed the power allocation scheme for the coverage optimization in different scenarios [4-8], such as femtocell networks [4], sensor networks [5, 6], and cellular networks $[7,8]$. Due to the fact that relay scheme do not need to increase the transmission power, it is suitable for the coverage extension in the shadow area. However, for the optimization of the wireless communication system, there are two main objects: the energy-saving and the interference minimization [9-11]. To ensure the QoE of users in the shadow area, the transmitters increase their transmission power wich will consume more energy for the coverage and bring a higher interference power from the adjacent cells. Moreover, the relay scheme for the coverage optimization needs more infrastructures which increase the cost of the network construction and increase the risk of security for entire networks $[12,13]$. Therefore, the optimization of the network coverage is still a challenging task.

Transformation electromagnetic theory brings a new view to understand the propagation process with the electromagnetic wave. It is based on the coordinate transformations operation on the Maxwell's equations which can be interpreted as the anisotropic compression and stretching of the constituent materials in the original space [14]. By the design of the materials interpretation, the propagation direction of electromagnetic wave can be controlled. The transformation electromagnetic theory is used for an invisibility cylindrical optical-cloak in flat space [15-17]. The arbitrary geometries cloak is designed by transformation electromagnetic technique in $[18,19]$. These results approved that the transformation electromagnetic theory has many advantages to design 
the cloak in the optical area. So, referring to the optical results, the transformation electromagnetic theory can be extended to be use in the ultra high frequency (UHF) bands. In [20], by using the transformation electromagnetic theory, the forward and/or backward scattering of the objects is reduced to improve the antenna performance at $2 \mathrm{GHz}$. Therefore, the transformation electromagnetic techniques can be used to design a special cloak (e.g., covering the surface of obstacles) to improve the signal coverage in the shadow area. Compared with the increasing the transmission power method or the relay-assisted scheme, the transformation electromagnetic techniques bring a new method for the coverage optimization which do not need to increase the transmission power or build more network infrastructures.

In this paper, in order to solve the coverage problem in the shadow area at UHF bands, a special cloak that covers the surface of obstacles is designed to improve the performance for the signal coverage performance in the shadow area. The main contributions are summarized as follows.

(i) An electromagnetic cloak is designed for improving the coverage performance of network. It can be used to strengthen the signal power in the shadow area without increasing the transmission power or using relay. It provides a new point of view for the coverage optimization.

(ii) Due to the fact that shapes of most obstacles are rectangular, the rectangular cloak is designed. In order to solve the calculation problem caused by the break point in the first-order derivative in the cylindrical coordinates system, the boundary functions of the rectangular cloak are approximately expressed by the Fourier fitting method. The discussion is extended to the gap-existed cloak situation.

(iii) The simulation results show that the strength of signal coverage will not be reduced after across the designed cloak. Therefore, the effectiveness of the coverage optimization method which is based on transformation electromagnetic theory is proved.

The rest of this paper is organized as follows. The transformation electromagnetic theory is introduced in Section 2. In Section 3, a rectangular special cloak is designed for coverage optimization. The related material properties of cloak are calculated by the transformation electromagnetic technique. The performance of rectangular cloak in current mobile bands is validated by the simulation in Section 4. Finally, the conclusions are presented in Section 5.

\section{Transformation Electromagnetic Theory for Coverage Optimization}

Due to the fact that one of the most common shapes of the obstacles is rectangular, we consider the case that the shadow area is caused by the blocking of the rectangular obstacle which is marked with the grid in Figure 1. The performance of the plane wave propagation is used to evaluate the improvement of the coverage. In the time-harmonic field, the electric field varies periodically and sinusoidally with

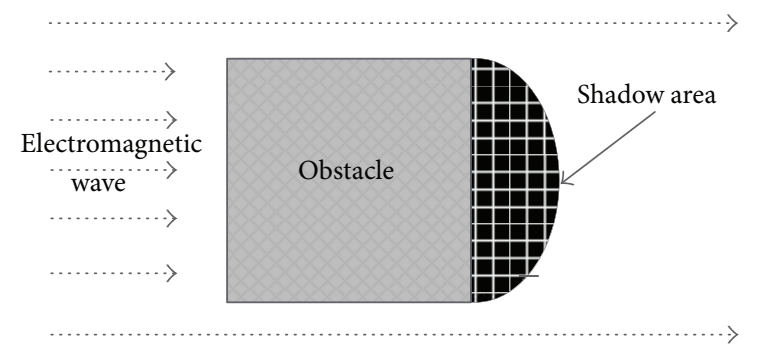

FIGURE 1: The obstacle blocks the electromagnetic waves and forms the shadow area.

time. The transformation electromagnetic theory provides a pathway to design the material parameters to control the electromagnetic wave propagation and improve the coverage reliability in the shadow area.

The transformation electromagnetic theory is based on two assumptions: the invariance of Maxwell's equations under coordinate transformations, and the material parameters in transformed coordinate system can be seen as a set of transformed material parameters in the original coordinate system [21].

In the time-harmonic field, the curl Maxwell's equations give the relationship between the electric field $\mathbf{E}$ and the magnetic field $\mathbf{H}$ [13]:

$$
\begin{aligned}
\nabla \times \mathbf{E} & =-j \omega \mu \mathbf{H}, \\
\nabla \times \mathbf{H} & =j \omega \varepsilon \mathbf{E}, \\
\mathbf{D} & =\varepsilon \mathbf{E}, \\
\mathbf{B} & =\mu \mathbf{H},
\end{aligned}
$$

where $\mathbf{B}$ denotes the magnetic flux densities, $\mathbf{D}$ denotes the electric flux densities, $\omega$ is angular frequency, $\mu$ denotes the electric-permittivity tensor, and $\varepsilon$ denotes the magneticpermeability tensor.

In the transformed coordinate system, Maxwell's equations have the same form as in the original coordinate system, which can be written as

$$
\begin{gathered}
\nabla \times \mathbf{E}^{\prime}=-j \omega \mu^{\prime} \mathbf{H}^{\prime}, \\
\nabla \times \mathbf{H}^{\prime}=j \omega \varepsilon^{\prime} \mathbf{E}^{\prime} .
\end{gathered}
$$

The material property tensors $\left(\mu^{\prime}\right.$ and $\left.\varepsilon^{\prime}\right)$ in the transformed coordinate system are derived from the $\mu$ and $\varepsilon$ tensor in the original space as follows:

$$
\begin{aligned}
\mu^{\prime} & =\frac{\mathbf{A} \mu \mathbf{A}^{T}}{\operatorname{det} \mathbf{A}}, \\
\varepsilon^{\prime} & =\frac{\mathbf{A} \varepsilon \mathbf{A}^{T}}{\operatorname{det} \mathbf{A}},
\end{aligned}
$$

where $\mathbf{A}$ is the transformation matrix from the original coordinate system to the transformed coordinate system and $\operatorname{det} \mathbf{A}$ represents the determinant of the transformation matrix $\mathbf{A}$. 
The transformation electromagnetic theory is based on the materials interpretation between the original coordinate system and the transformed coordinate system. Since the propagation characteristics in the media are related to the material parameters, designing the proper material parameters by the transformation electromagnetic theory can control the radio propagation in the space. Controlling with the propagation direction of electromagnetic wave by material brings a new way to consider the coverage optimization in the wireless network.

Generally speaking, the transformation electromagnetic design procedure can be summarized as follows.

(i) Determine the model of transmission with electromagnetic wave in original coordinate system. Usually choose a plane wave propagation in the free space.

(ii) Set a volume of space in the original coordinate system and the associated volume of space in the transformed coordinate system.

(iii) Define the coordinate transformation from the original space to the transformed space.

(iv) Calculate the material parameters in transformed space (the space of the cloak) via (3).

(v) Interpret the material parameters in the original space, and obtain the desired propagation results (improve the coverage in the shadow area).

\section{Design Rectangular Cloak via Transformation Electromagnetic Theory}

The aim of the coverage optimization in networks is minimizing the transmission power and enlarging the coverage area (including the shadow area). In this section, a special cloak that covers the obstacle is designed to improve the coverage in the shadow area. Due to the fact that shapes of most obstacles are rectangular, the rectangular cloak is designed in this paper. It is hard to calculate the material parameters at the break point of the first-order derivative in the cylindrical coordinates system. Fortunately, the boundary functions of rectangular cloak can be expressed by calculating the approximated Fourier series boundary functions by the Fourier series fitting method. The transformed material parameters (including the electric-permittivity tensor and the magneticpermeability tensor) are calculated by transformation electromagnetic theory by using the approximated boundary functions. The detail of design is described as the following parts.

The cylindrical coordinate system is proper to describe the original and the transformed spaces. As shown in Figure 2, the coordinate system transformation is between the original coordinate system $(\rho, \theta, z)$ and transformed coordinate system $\left(\rho^{\prime}, \theta^{\prime}, z^{\prime}\right)$.

The transformed electric-permittivity tensor and the magnetic-permeability tensor are denoted by $\varepsilon^{\prime}$ and $\mu^{\prime}$, respectively. The electric-permittivity tensor $\varepsilon^{\prime}$ is transformed from $\varepsilon$. Usually, the magnetic-permeability tensor $\mu^{\prime}$ equals

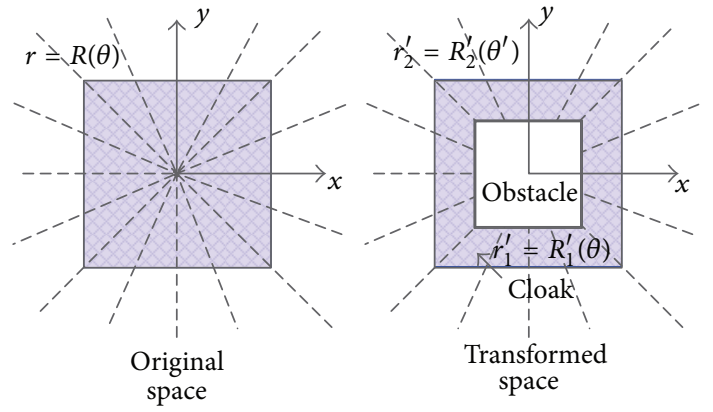

FIGURE 2: The generalized coordinate transformation.

electric-permittivity $\varepsilon^{\prime}$. So, $\varepsilon^{\prime}$ is considered in the following analysis. The transformed electric-permittivity tensor $\varepsilon^{\prime}$ is

$$
\varepsilon^{\prime}=\left(\begin{array}{lll}
\varepsilon_{x^{\prime} x^{\prime}} & \varepsilon_{x^{\prime} y^{\prime}} & \varepsilon_{x^{\prime} z^{\prime}} \\
\varepsilon_{y^{\prime} x^{\prime}} & \varepsilon_{y^{\prime} y^{\prime}} & \varepsilon_{y^{\prime} z^{\prime}} \\
\varepsilon_{z^{\prime} x^{\prime}} & \varepsilon_{z^{\prime} y^{\prime}} & \varepsilon_{z^{\prime} z^{\prime}}
\end{array}\right) .
$$

In the cylindrical coordinate system, the original space $r \leq R(\theta)$ is transformed into the transformed space (cloak) $R_{1}^{\prime}\left(\theta^{\prime}\right) \leq r^{\prime} \leq R_{2}^{\prime}\left(\theta^{\prime}\right)$, where $R_{1}^{\prime}\left(\theta^{\prime}\right)$ and $R_{2}^{\prime}\left(\theta^{\prime}\right)$ denote the inner and the outer boundary function of cloak, respectively [18]. By the coordinate transformation, the original space $r \leq$ $R(\theta)$ is transformed into the transformed space which can be described as

$$
r^{\prime}=R_{1}^{\prime}(\theta)+\frac{R_{2}^{\prime}(\theta)-R_{1}^{\prime}(\theta)}{R_{2}^{\prime}(\theta)} r, \quad \theta^{\prime}=\theta, z^{\prime}=z .
$$

The values of the transformed permittivity tensor for twodimensional (2D) cloak are calculated mathematically as follows:

$$
\begin{aligned}
\varepsilon_{x^{\prime} x^{\prime}}^{\prime}= & \left(\left\{\left[r^{\prime}-R_{1}^{\prime}\left(\theta^{\prime}\right)\right]^{2}+U^{2}\right\} \cos ^{2} \theta^{\prime}\right. \\
& \left.-2 U r^{\prime} \sin \theta^{\prime} \cos \theta^{\prime}+r^{\prime 2} \sin ^{2} \theta^{\prime}\right) \\
\times & \left(r^{\prime}\left[r^{\prime}-R\left(\theta^{\prime}\right)\right]\right)^{-1}, \\
\varepsilon_{x^{\prime} y^{\prime}}^{\prime}= & \varepsilon_{y^{\prime} x^{\prime}} \\
= & \left(\left\{U^{2}-R_{1}^{\prime}\left(\theta^{\prime}\right)\left[2 r^{\prime}-R_{1}^{\prime}\left(\theta^{\prime}\right)\right]\right\} \sin \theta^{\prime} \cos \theta^{\prime}\right. \\
+ & \left.U r^{\prime}\left(\cos ^{2} \theta^{\prime}-\sin ^{2} \theta^{\prime}\right)\right) \\
\times & \left(r^{\prime}\left[r^{\prime}-R_{1}^{\prime}\left(\theta^{\prime}\right)\right]\right)^{-1},
\end{aligned}
$$




$$
\begin{aligned}
& \varepsilon_{y^{\prime} y^{\prime}}^{\prime}=\left(\left\{\left[r^{\prime}-R_{1}^{\prime}\left(\theta^{\prime}\right)\right]^{2}+U^{2}\right\} \sin ^{2} \theta^{\prime}\right. \\
&\left.+2 U r^{\prime} \sin \theta^{\prime} \cos \theta^{\prime}+r^{\prime 2} \cos ^{2} \theta^{\prime}\right) \\
& \times\left(r\left[r^{\prime}-R_{1}^{\prime}\left(\theta^{\prime}\right)\right]\right)^{-1}, \\
& \varepsilon_{z^{\prime} z^{\prime}}^{\prime}= \frac{r^{\prime}-R_{1}^{\prime}\left(\theta^{\prime}\right)}{r^{\prime}}\left[\frac{R_{2}^{\prime}\left(\theta^{\prime}\right)}{R_{2}^{\prime}\left(\theta^{\prime}\right)-R_{1}^{\prime}\left(\theta^{\prime}\right)}\right]^{2}, \\
& \varepsilon_{x^{\prime} z^{\prime}}^{\prime}=\varepsilon_{y^{\prime} z^{\prime}}^{\prime}=\varepsilon_{z^{\prime} x^{\prime}}^{\prime}=\varepsilon_{z^{\prime} y^{\prime}}^{\prime}=0,
\end{aligned}
$$

where

$$
\begin{aligned}
U= & \left(\left[r^{\prime}-R_{1}^{\prime}\left(\theta^{\prime}\right)\right] R_{1}^{\prime}\left(\theta^{\prime}\right)\left(d R_{2}^{\prime}\left(\theta^{\prime}\right) / d \theta^{\prime}\right)\right. \\
& \left.-\left[r^{\prime}-R_{2}^{\prime}\left(\theta^{\prime}\right)\right]\left(d R_{1}^{\prime}\left(\theta^{\prime}\right) / d \theta^{\prime}\right) R_{2}^{\prime}\left(\theta^{\prime}\right)\right) \\
& \times\left(R_{2}^{\prime}\left(\theta^{\prime}\right)\left[R_{2}^{\prime}\left(\theta^{\prime}\right)-R_{1}^{\prime}\left(\theta^{\prime}\right)\right]\right)^{-1} .
\end{aligned}
$$

The $d R_{1}^{\prime}\left(\theta^{\prime}\right) / d \theta^{\prime}$ and $d R_{2}^{\prime}\left(\theta^{\prime}\right) / d \theta^{\prime}$ denote the first-order derivative of $R_{1}^{\prime}(\theta)$ and $R_{2}^{\prime}(\theta)$, respectively. When the firstorder derivative of boundary function is continuous, the electric-permittivity tensor and the magnetic-permeability tensor of the cloak can be solved by (6)-(7). In fact, in wireless communication environments, many shapes do not have the first-order continual derivative of boundary function at the sharp corners (such as triangle, rectangle, and pentagon). These kinds of mathematical functions cannot be solved when calculating the results at the break point of the first-order derivative.

In traditional cellular communication scenarios, the rectangular shape is one of the most common shapes of the obstacle. Since the cloak covers the rectangular obstacle, the cloak is like a rectangular ring. The inner boundary and the outer boundary can be described as

$$
\begin{aligned}
R_{1}^{\prime}\left(\theta^{\prime}\right)=\left\{\begin{array}{cl}
\frac{a}{2 \cos \theta^{\prime}}, & \theta^{\prime} \in\left(-\frac{\pi}{4}, \frac{\pi}{4}\right), \\
\frac{a}{2 \sin \theta^{\prime}}, & \theta^{\prime} \in\left(\frac{\pi}{4}, \frac{3 \pi}{4}\right), \\
-\frac{a}{2 \cos \theta^{\prime}}, & \theta^{\prime} \in\left(\frac{3 \pi}{4}, \frac{5 \pi}{4}\right), \\
-\frac{a}{2 \sin \theta^{\prime}}, & \theta^{\prime} \in\left(\frac{5 \pi}{4}, \frac{7 \pi}{4}\right),
\end{array}\right. \\
R_{2}^{\prime}\left(\theta^{\prime}\right)=\left\{\begin{array}{cl}
\frac{b}{2 \cos \theta^{\prime}}, & \theta^{\prime} \in\left(-\frac{\pi}{4}, \frac{\pi}{4}\right), \\
\frac{b}{2 \sin \theta^{\prime}}, & \theta^{\prime} \in\left(\frac{\pi}{4}, \frac{3 \pi}{4}\right), \\
-\frac{b}{2 \cos \theta^{\prime}}, & \theta^{\prime} \in\left(\frac{3 \pi}{4}, \frac{5 \pi}{4}\right), \\
-\frac{b}{2 \sin \theta^{\prime}}, & \theta^{\prime} \in\left(\frac{5 \pi}{4}, \frac{7 \pi}{4}\right),
\end{array}\right.
\end{aligned}
$$

where $a$ and $b$ denote the side length of the inner rectangular boundary and the outer rectangular boundary, respectively.

There are four break points where the first-order derivative of the boundary is discontinued. Take the square shape as an example, the value of $\left(d R_{1}^{\prime}\left(\theta^{\prime}\right)\right) / d \theta^{\prime}$ and $\left(d R_{2}^{\prime}\left(\theta^{\prime}\right)\right) / d \theta^{\prime}$ with cloak inner boundary and outer boundary are discontinued when $\theta=\theta^{\prime}=((2 n-1) \pi) / 4, n \in N, \theta \in[0,2 \pi)$, and $\theta^{\prime} \in[0,2 \pi)$. It is hard to get the value of electric-permittivity $\varepsilon_{x^{\prime} x^{\prime}}, \varepsilon_{x^{\prime} y^{\prime}}, \varepsilon_{y^{\prime} x^{\prime}}, \varepsilon_{y^{\prime} y^{\prime}}, \varepsilon_{z^{\prime} z^{\prime}}$.

In order to solve this calculation problems, the inner boundary function $R_{1}^{\prime}\left(\theta^{\prime}\right)$ and the outer boundary function $R_{2}^{\prime}\left(\theta^{\prime}\right)$ of the cloak can be replaced by the Fourier approximated continuous functions with period $2 \pi$. The Fourier series can be described as

$$
f\left(\theta^{\prime}\right)=a_{0}+\sum_{n=1}^{\infty}\left(a_{n} \cos n \theta^{\prime}+b_{n} \sin n \theta^{\prime}\right)
$$

where $a_{n}$ and $b_{n}$ are the Fourier series coefficients.

The Fourier series coefficients of the approximated boundary functions is determined by the least-square-fitting method. A number of the feature points (must include the break points, $\theta^{\prime}=n \pi / 4, n \in N$ ) are used to describe the boundary shape as shown in Figure 3 . After using the least-square-fitting method, the $a_{n}$ and $b_{n}$ are determined for the approximate boundary functions. The $f_{1}\left(\theta^{\prime}\right)$ and $f_{2}\left(\theta^{\prime}\right)$ denote the inner boundary and the outer boundary, respectively. The first-order derivatives of boundary functions $\left(f_{1}\left(\theta^{\prime}\right)\right.$ and $\left.f_{2}\left(\theta^{\prime}\right)\right)$ are the continuous functions. In Figure 4 , it shows approximated boundaries of cloak which are described by $f_{1}\left(\theta^{\prime}\right)$ and $f_{2}\left(\theta^{\prime}\right)$. Then by substituting the approximated boundary functions $f_{1}\left(\theta^{\prime}\right)$ and $f_{2}\left(\theta^{\prime}\right)$ into the (6)-(7), the material parameters of the cloak can be calculated.

Based on the previous calculation, the calculation flowchart is shown in the Figure 5. This designing method is limited not only to the rectangular cloak but also for all the shapes which have the discontinuous boundary functions in first-order derivative.

\section{Electric Field Performances with the Rectangular Cloak in Wireless Communication Frequency}

The rectangular cloak performances are evaluated in UHF bands. The radio frequency is chosen as $900 \mathrm{MHz}$. Since the obstacles are usually far from the base station in the cellular networks, the propagation process of electromagnetic wave can be seen as a plane wave transmission process. The cloak is assumed to cover the obstacle to enhance the coverage in shadow area, so the inner boundary of cloak has the same boundary function with the obstacle. Due to the fact that cloak performance is not related to the size of the obstacle, to facilitate processing with simulation, a small square obstacle is chosen as an example. The inner and the outer boundary functions of the cloak are denoted by $R_{1}^{\prime}\left(\theta^{\prime}\right)$ and $R_{2}^{\prime}\left(\theta^{\prime}\right)$.

The size of obstacle is $0.2 \mathrm{~m} \times 0.2 \mathrm{~m}$. The side length of the inner boundary and the outer boundary of cloak are $0.2 \mathrm{~m}$ 


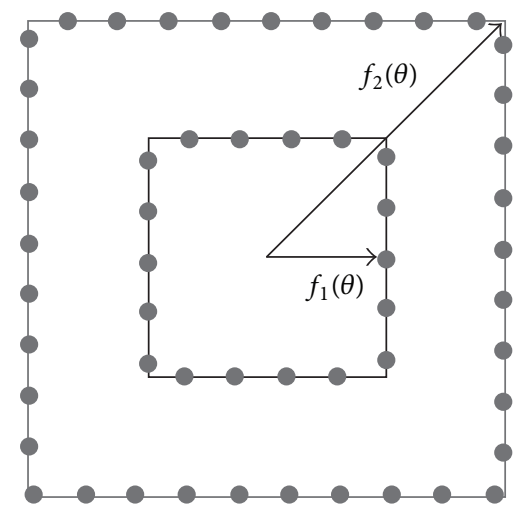

Figure 3: A large number of the feature points describe the boundary functions.

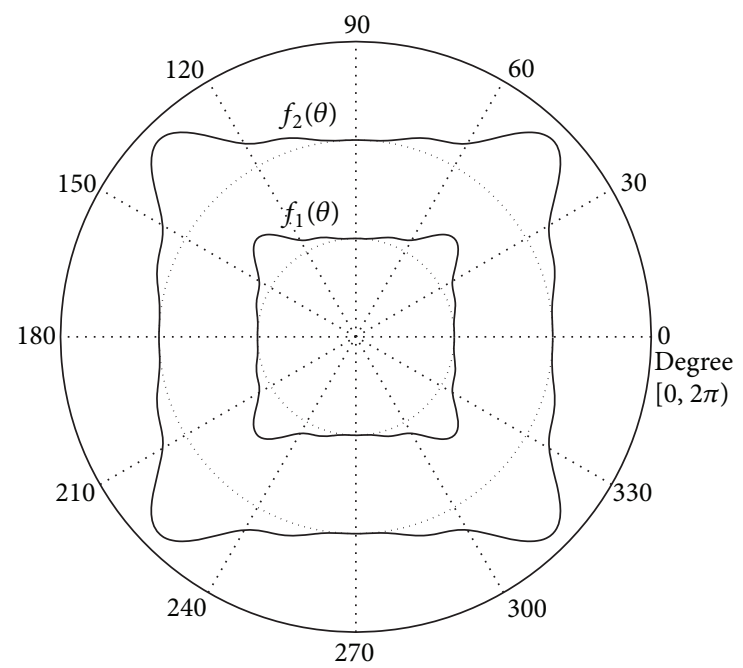

FIgURE 4: The approximate boundaries of the cloak.

and $0.4 \mathrm{~m}$, respectively. The cloak boundary functions can be expressed as

$$
\begin{aligned}
R_{1}^{\prime}\left(\theta^{\prime}\right)=\left\{\begin{array}{cl}
\frac{0.2}{2 \cos \theta^{\prime}}, & \theta^{\prime} \in\left(-\frac{\pi}{4}, \frac{\pi}{4}\right), \\
\frac{0.2}{2 \sin \theta^{\prime}}, & \theta^{\prime} \in\left(\frac{\pi}{4}, \frac{3 \pi}{4}\right), \\
-\frac{0.2}{2 \cos \theta^{\prime}}, & \theta^{\prime} \in\left(\frac{3 \pi}{4}, \frac{5 \pi}{4}\right), \\
-\frac{0.2}{2 \sin \theta^{\prime}}, & \theta^{\prime} \in\left(\frac{5 \pi}{4}, \frac{7 \pi}{4}\right),
\end{array}\right. \\
R_{2}^{\prime}\left(\theta^{\prime}\right)=\left\{\begin{array}{cl}
\frac{0.4}{2 \cos \theta^{\prime}}, & \theta^{\prime} \in\left(-\frac{\pi}{4}, \frac{\pi}{4}\right), \\
\frac{0.4}{2 \sin \theta^{\prime}}, & \theta^{\prime} \in\left(\frac{\pi}{4}, \frac{3 \pi}{4}\right), \\
-\frac{0.4}{2 \cos \theta^{\prime}}, & \theta^{\prime} \in\left(\frac{3 \pi}{4}, \frac{5 \pi}{4}\right), \\
-\frac{0.4}{2 \sin \theta^{\prime}}, & \theta^{\prime} \in\left(\frac{5 \pi}{4}, \frac{7 \pi}{4}\right) .
\end{array}\right.
\end{aligned}
$$

There are four break points in the first-order derivative of boundary functions (both $R_{1}^{\prime}$ and $R_{2}^{\prime}$ ) when $\theta^{\prime}=n \pi / 4$ $(n=1,2,3, \ldots)$. After using the Fourier least-square-fitting method, the approximate boundary functions of cloak are calculated as

$$
\begin{aligned}
R_{1}^{\prime}\left(\theta^{\prime}\right) \approx & f_{1}\left(\theta^{\prime}\right) \\
= & 0.4512-0.06732 \cos 4 \theta^{\prime}+0.02494 \cos 8 \theta^{\prime} \\
& -0.01553 \cos 12 \theta^{\prime}+0.006722 \cos 16 \theta^{\prime}, \\
R_{2}^{\prime}\left(\theta^{\prime}\right) \approx & f_{2}\left(\theta^{\prime}\right) \\
= & 0.2256-0.03366 \cos 4 \theta^{\prime}+0.01247 \cos 8 \theta^{\prime} \\
& -0.007764 \cos 12 \theta^{\prime}+0.003361 \cos 16 \theta^{\prime} .
\end{aligned}
$$

Finally, the material parameters of cloak $\left(\varepsilon^{\prime}\right.$ and $\left.\mu^{\prime}\right)$ can be calculated via (6)-(7).

As shown in Figure 6, the transverse-electric (TE) plane wave transmits from the left to the right with unit amplitude. The signal strength is decreased at the back side of obstacle.

The coverage performance with cloaked obstacle is shown in Figure 7. Compared with the performance in noncloak situation, the signal strength in the shadow area with cloaked obstacle is improved. The electromagnetic wave is smoothly guided by the cloak and transmitted into the shadow area. It shows a more stable and predictable coverage performance in the shadow area.

Due to the fact that cloak cannot always keep perfectly all the time, the imperfect situation that the cloak has a gap should be considered as well. In Figure 8, there is a gap in the cloak whose width is $0.1 \mathrm{~m}$. The TE plane waves transmit from the left to the right. It can be simply described as a situation that the TE plane waves transmit across a semi-infinite space which is shown in Figure 8. The electric-permittivity and magnetic-permeability in medium I and medium II are $\varepsilon_{1}^{\prime \prime}, \mu_{1}^{\prime \prime}$ and $\varepsilon_{2}^{\prime \prime}, \mu_{2}^{\prime \prime}$, respectively. The thickness of the middle medium material is $d$, and the material properties are described as $\varepsilon^{\prime \prime}$, $\mu^{\prime \prime}$. When the TE plane electromagnetic waves transmit from left to right ( $+\widehat{z}$ direction, from medium I to medium II), the reflection occurs at the place of two boundaries $z=0$ and $z=$ $d$. The transmission coefficient is always less than 1 [22] which means that only a part of electromagnetic waves can transmit into the medium II. When electromagnetic waves pass through the gap, a part of electromagnetic waves are blocked. As shown in Figure 9, the signal coverage performance in the shadow area is deteriorated slightly because a part of signal power is leaked into the surrounding space at the gap area.

As shown in Figure 10, the strength of the electromagnetic wave in the shadow area is compared. When the obstacle covers with the designed cloak, the signal power in the shadow area is more stable and stronger than in the case without cloaked obstacle situation. And when there is a gap on the cloak, the performance of cloak is still acceptable. The simulation results prove that the designed cloak can improve the performance of the coverage in the shadow area. 


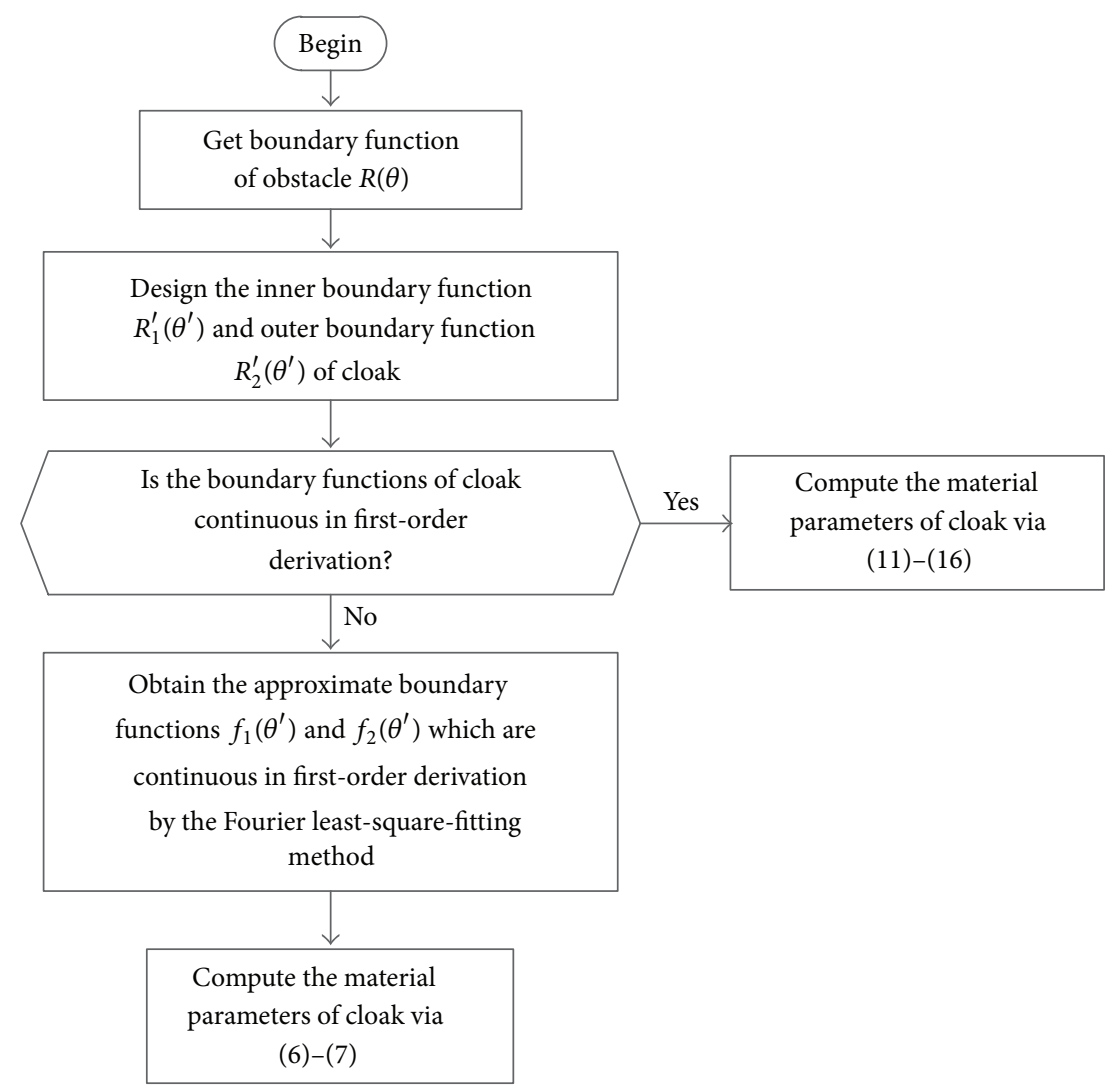

FIGURE 5: The calculation flowchart of the material parameters for the cloak.

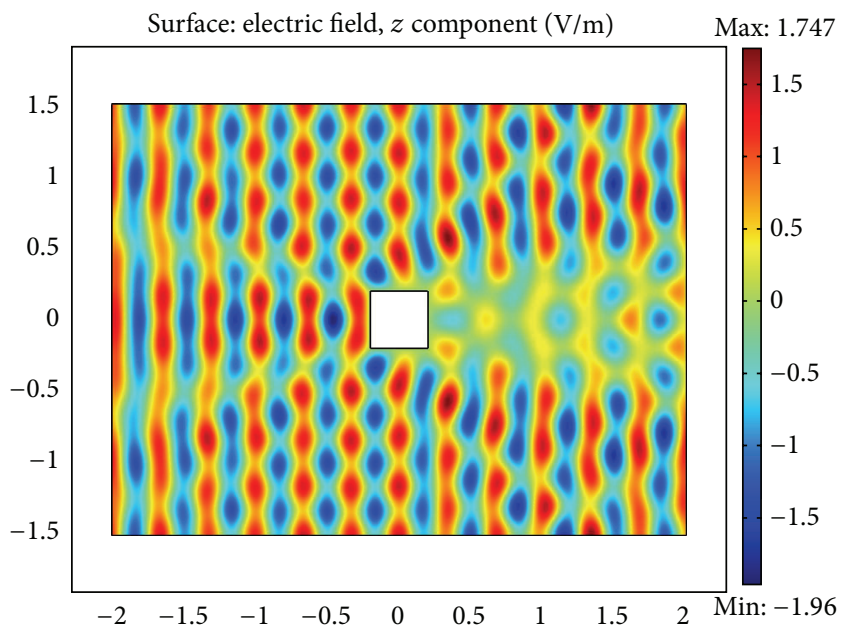

Figure 6: The coverage performance when the obstacle block the electromagnetic wave.

\section{Conclusion}

This paper presents a special rectangular cloak design for the improvement of the signal coverage in wireless communications. In order to solve the calculation problem with the rectangular shape in the electromagnetic transformation theory, the approximated boundary functions which use the the

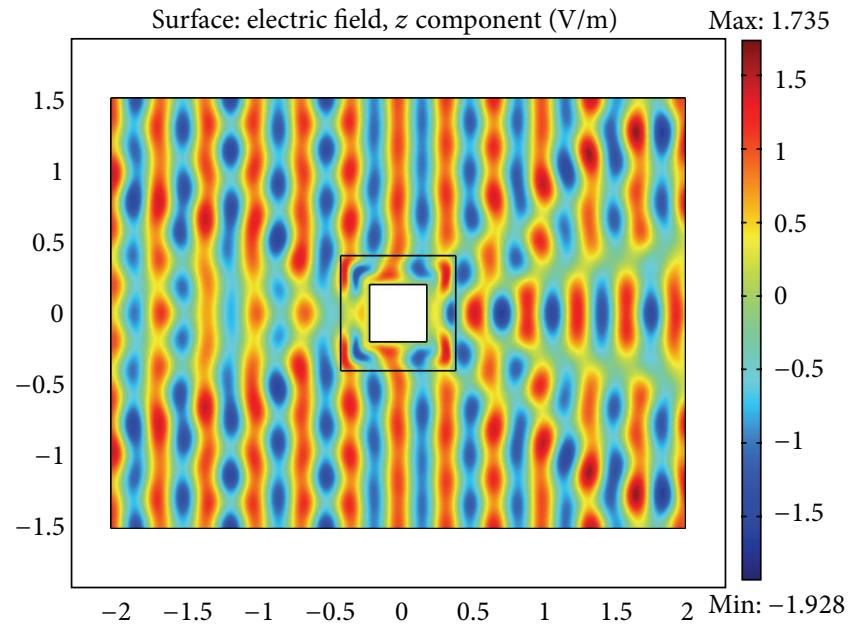

FIGURE 7: The coverage performance when using the cloak to enhance the signal strength in the shadow area.

Fourier least-square-fitting method to approximate describe the rectangular boundary function of the rectangular cloak are proposed. The simulation results prove that the designed cloak can improve the coverage performance in the shadow area effectively. In addition, the gap-existed cloak also brings an acceptable performance in the shadow area. Compared with the transmission power optimizing or relay-assisted 


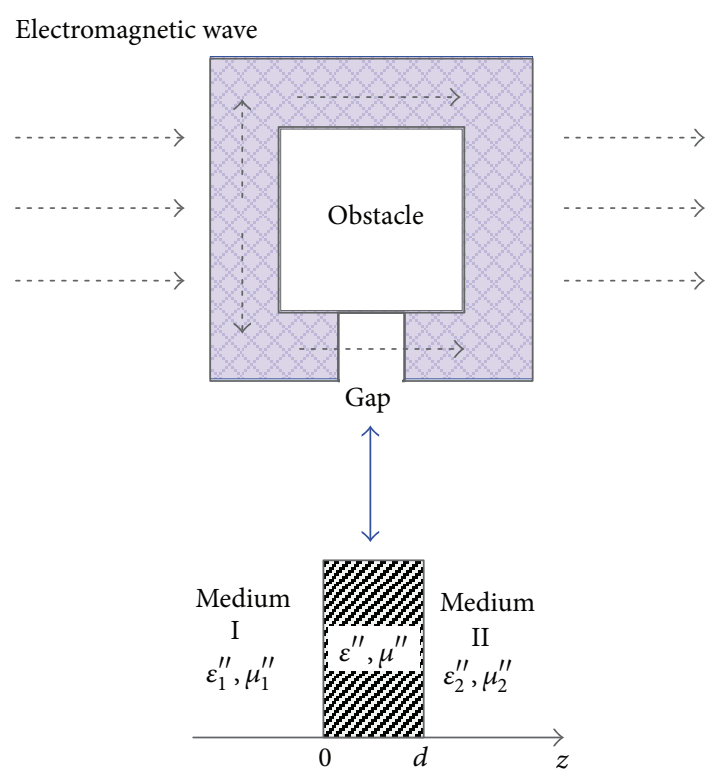

FIGURE 8: The situation of gap in the bottom of cloak.

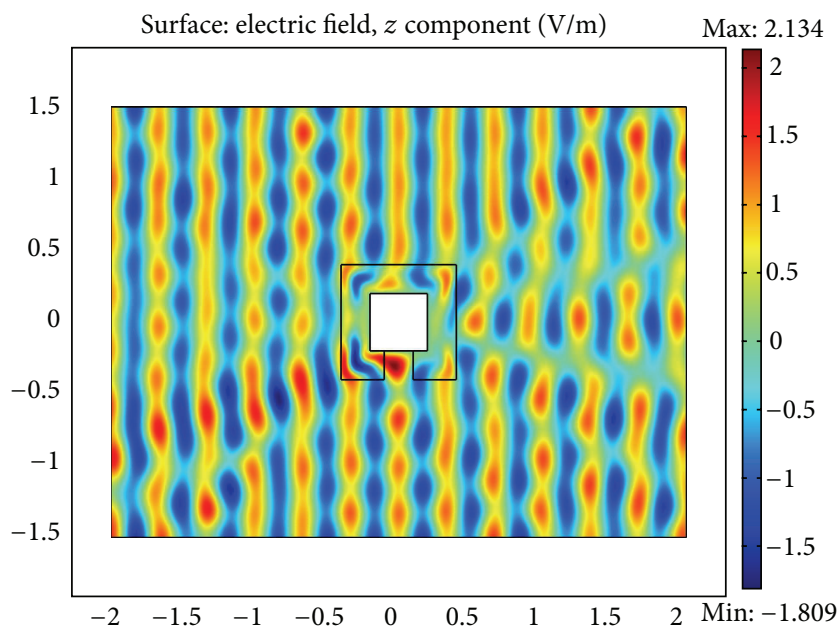

FIGURE 9: The coverage performance with the gap-existed cloak situation.

scheme, the transformation electromagnetic theory provides a new viewpoint and gives an efficient tool for the coverage optimization in the shadow area.

\section{Acknowledgments}

This work is supported by the Fundamental Research Funds for the Central Universities under Grant nos. 2012YJS017 and 2010JBZ008, Key Project of State Key Lab of Rail Traffic and Control under Grant nos. RCS2012ZZ004 and RCS2011ZZ008, the Key Grant Project of Chinese Ministry of Education no. 313006, the National Natural Science Foundation of China under Grant no. 61222105, the Natural Science Foundation of China under Grant no. U1334202, and

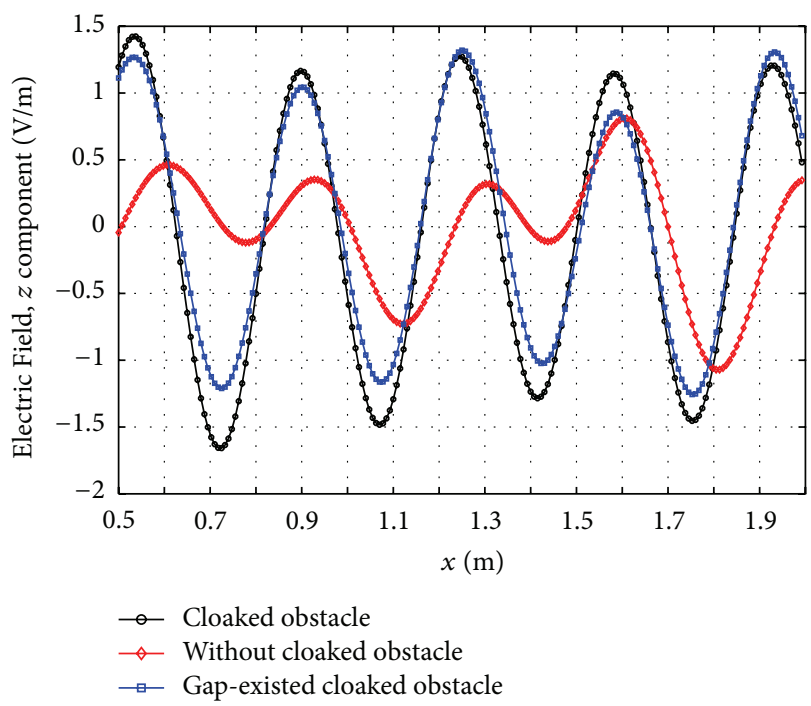

FIGURE 10: The comparison of signal strength in the shadow area.

Program for New Century Excellent Talents in University under Grant no. NCET-09-0206.

\section{References}

[1] M. Pätzold, Mobile Fading Channels: Modelling, Analysis, Simulation, Wiley, New York, NY, USA, 2002.

[2] T. S. Rappaport, Wireless Communications: Principles and Practice, Prentice-Hall, Endlewood Cliffs, NJ, USA, 2nd edition, 2001.

[3] A. Goldsmith, Wireless Communications, Cambridge University Press, New York, NY, USA, 2005.

[4] I. Ashraf, H. Claussen, and L. T. W. Ho, "Distributed radio coverage optimization in enterprise femtocell networks," in Proceedings of the IEEE International Conference on Communications (ICC '10), May 2010.

[5] Z. Guoping, K. Sivanand, F. Chin, and C. C. Ko, "Quality of service and coverage optimization in wireless sensor networks," in Proceedings of the 67th IEEE Vehicular Technology Conference (VTC '08), pp. 2824-2828, May 2008.

[6] J. Habibi, H. Mahboubi, and A. G. Aghdam, "A nonlinear optimization approach to coverage problem in mobile sensor networks," in Proceedings of the IEEE Conference on Decision and Control and European Control Conference (CDC-ECC '11), pp. 7255-7261, December 2011.

[7] D. Fagen, P. A. Vicharelli, and J. Weitzen, "Automated coverage optimization in wireless networks," in Proceedings of the 64th IEEE Vehicular Technology Conference (VTC '06), pp. 1043-1047, September 2006.

[8] R. Atawia, M. El Azab, T. Elshabrawy, and A. Mohamed, "Ranked overlapping coverage based construction of efficient neighboring cell list for GSM/UMTS cellular networks," in International Conference on Communications and Information Technology (ICCIT '12), pp. 254-259, June 2012.

[9] X. Cheng, Q. Yao, M. Wen, C.-X. Wang, L. Song, and B. Jiao, "Wideband channel modeling and ICI cancellation for vehicleto-vehicle communication systems," IEEE Journal on Selected Areas in Communnication, vol. 31, no. 9, pp. 434-448, 2013. 
[10] X. Cheng, C.-X. Wang, H. Wang et al., "Cooperative MIMO channel modeling and multi-link spatial correlation properties," IEEE Journal on Selected Areas in Communications, vol. 30, no. 2, pp. 388-396, 2012.

[11] X. Cheng, C.-X. Wang, D. I. Laurenson, S. Salous, and A. V. Vasilakos, "An adaptive geometry-based stochastic model for non-isotropic MIMO mobile-to-mobile channels," IEEE Transactions on Wireless Communications, vol. 8, no. 9, pp. 48244835, 2009.

[12] L. Xiao, T. E. Fuja, and D. J. Costello Jr., "An analysis of mobile relaying for coverage extension," in Proceedings of the IEEE International Symposium on Information Theory (ISIT '08), pp. 2262-2266, July 2008.

[13] Ö. Bulakci, S. Redana, B. Raaf, and J. Hämäläinen, "Impact of power control optimization on the system performance of relay based LTE-Advanced heterogeneous networks," Journal of Communications and Networks, vol. 13, no. 4, pp. 345-360, 2011.

[14] J. B. Pendry, D. Schurig, and D. R. Smith, "Controlling electromagnetic fields," Science, vol. 312, no. 5781, pp. 1780-1782, 2006.

[15] D. Schurig, J. B. Pendry, and D. R. Smith, "Calculation of material properties and ray tracing in transformation media," Optics Express, vol. 14, no. 21, 2006.

[16] S. A. Cummer, B.-I. Popa, D. Schurig, D. R. Smith, and J. Pendry, "Full-wave simulations of electromagnetic cloaking structures," Physical Review E, vol. 74, no. 3, Article ID 036621, 2006.

[17] M. Yan, Z. Ruan, and M. Qiu, "Cylindrical invisibility cloak with simplified material parameters is inherently Visible," Physical Review Letters, vol. 99, no. 23, Article ID 233901, 2007.

[18] C. Li, K. Yao, and F. Li, “Two-dimensional electromagnetic cloaks with non-conformal inner and outer boundaries," Optics Express, vol. 16, no. 23, pp. 19366-19374, 2008.

[19] W. X. Jiang, J. Y. Chin, Z. Li, Q. Cheng, R. Liu, and T. J. Cui, "Analytical design of conformally invisible cloaks for arbitrarily shaped objects," Physical Review E, vol. 77, no. 6, Article ID 066607, 2008.

[20] D.-H. Kwon and D. H. Werner, "Restoration of antenna parameters in scattering environments using electromagnetic cloaking," Applied Physics Letters, vol. 92, no. 11, Article ID 113507, 3 pages, 2008.

[21] D.-H. Kwon and D. H. Werner, "Transformation electromagnetics: an overview of the theory and applications," IEEE Antennas and Propagation Magazine, vol. 52, no. 1, pp. 24-46, 2010.

[22] D. Su, A. Cheng, and Sh. Xie, Electromagnetic Field and Electromagnetic Wave, Higher Education Press, Beijing, China, 2009. 

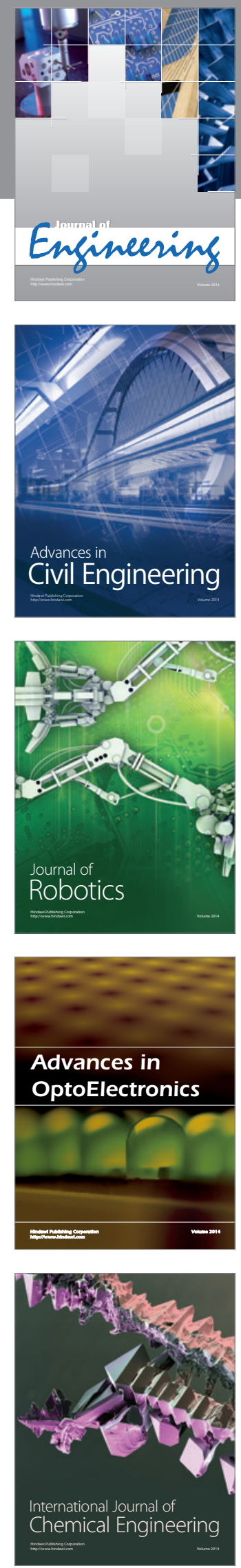

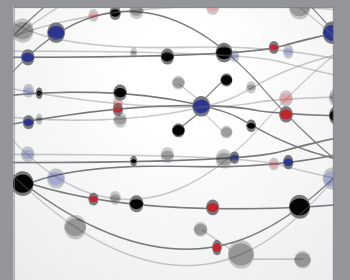

The Scientific World Journal
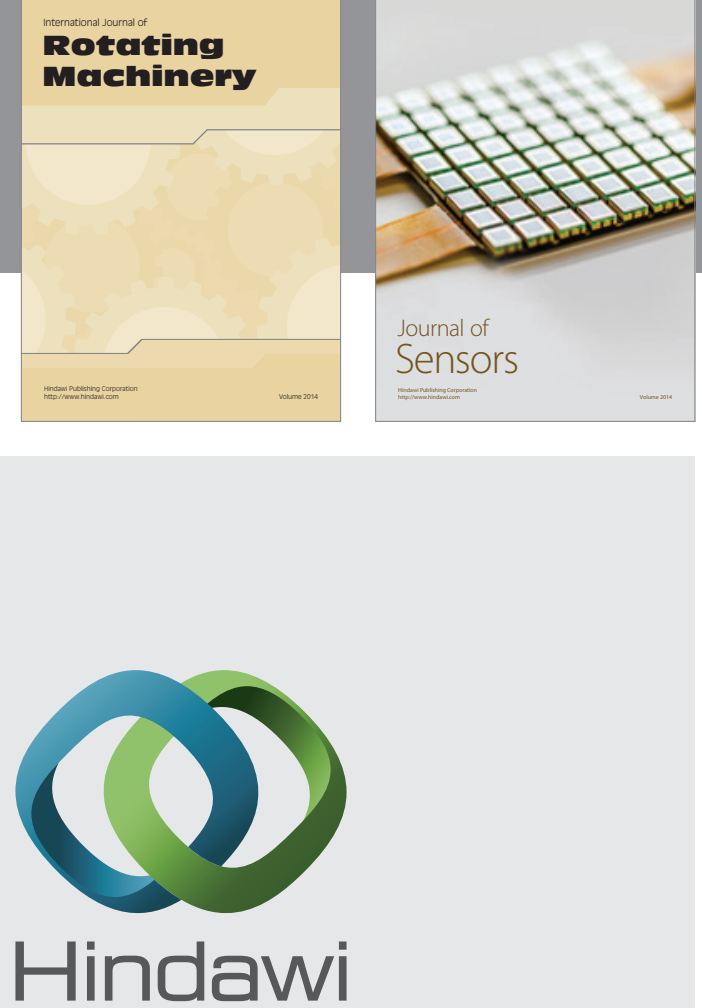

Submit your manuscripts at http://www.hindawi.com
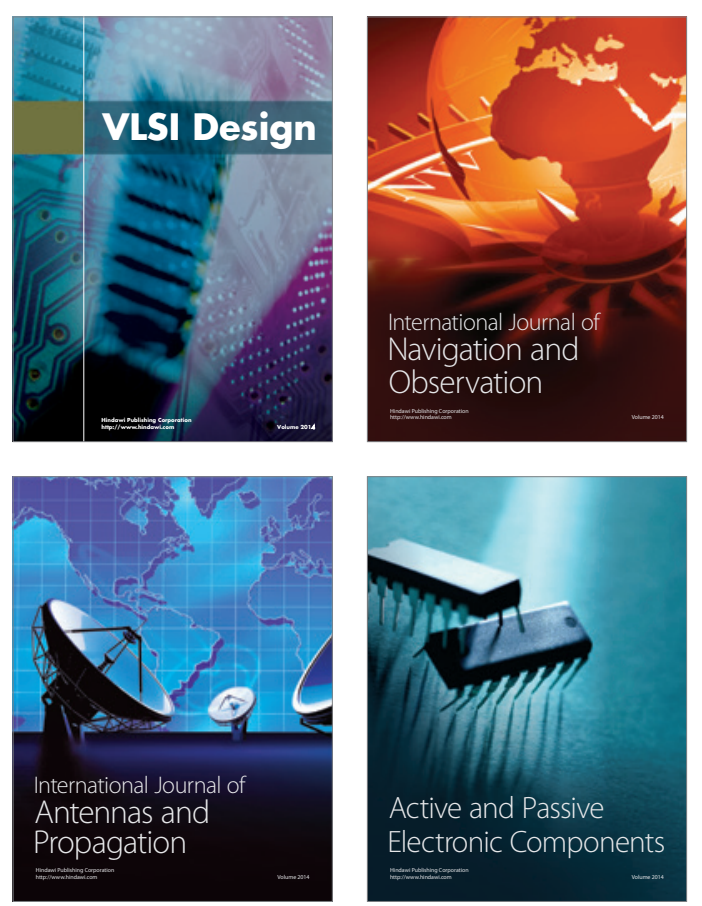
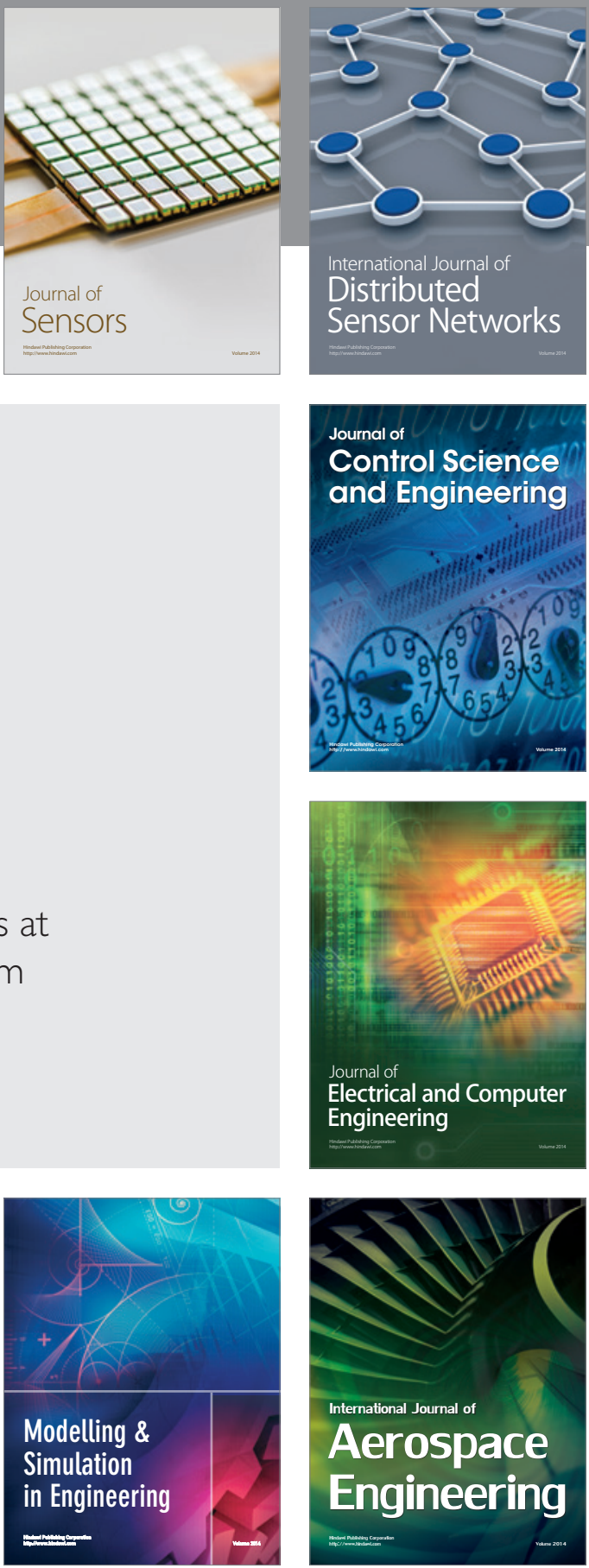

Journal of

Control Science

and Engineering
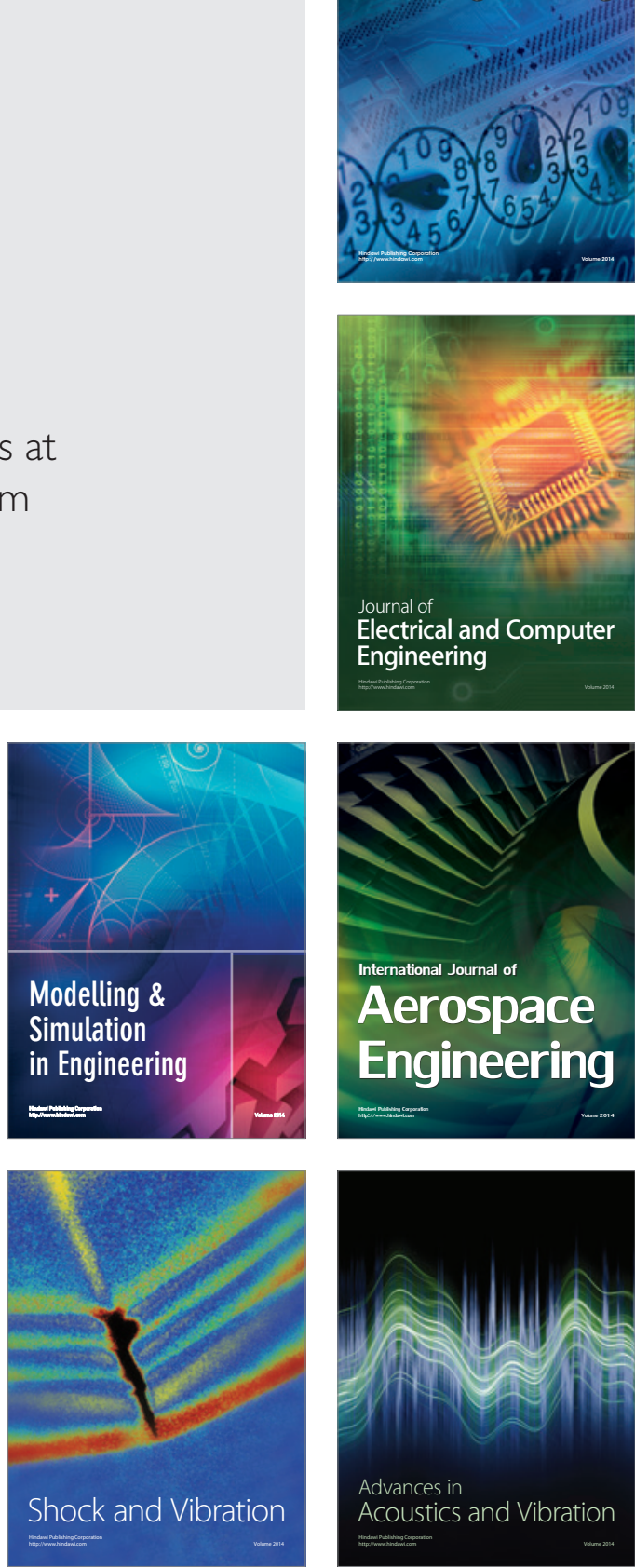